\title{
THE KERNEL OF A RULE OF APPROXIMATE INTEGRATION
}

\author{
J. H. LOXTON and J. W. SANDERS
}

(Received 4 September 1978)

\begin{abstract}
It is well known that the trapezoidal rule of quadrature is exact for linear functions on $[0,1]$, and easy to see that it is exact for functions of the form $f=l+g$ where $l$ is linear and $g$ is odd about $\frac{1}{2}$. Not so well known is an example of a continuous function for which the trapezoidal rule is exact but which does not have this form. In this paper we show that if the trapezoidal rule is exact for $f$ then $f$ has the form above provided it has either absolutely convergent Fourier series or continuous second derivative. We consider one-sided versions in which the approximate integrals are non-negative, and also characterize those sequences arising as the approximate integrals of a function with absolutely convergent Fourier series.
\end{abstract}

\section{Introduction and notation}

We write $\mathscr{T}$ for the trapezoidal rule of quadrature whose $n$th approximate integral (for $n=1,2,3, \ldots$ ) assigns to any continuous function $f$ on $[0,1]$ the value

$$
T_{n} f=n^{-1}\left(\frac{1}{2} f(0)+\sum_{j=1}^{n-1} f(j / n)+\frac{1}{2} f(1)\right)
$$

It is well known that, when $f^{\prime \prime}$ is continuous, given any $n$ there exists $\xi \in[0,1]$ for which

$$
\int_{0}^{1} j=T_{n} f-f^{\prime \prime}(\xi) /\left(12 n^{2}\right)
$$

In particular, $\mathscr{T}$ is exact for linear functions. However, $\mathscr{T}$ is also exact for other functions: whenever $g$ is odd about the point $\frac{1}{2}$,

$$
\int_{0}^{1} g=0=T_{n} g \text { for } n=1,2,3, \ldots,
$$

(C) Copyright Australian Mathematical Society 1980

Copyright. Apart from any fair dealing for scholarly purposes as permitted under the Copyright Act, no part of this JOURNAL may be reproduced by any process without written permission from the Treasurer of the Australian Mathematical Society. 
since the points of evaluation of $\mathscr{T}$ are symmetric about $\frac{1}{2}$. So $\mathscr{T}$ is exact for any sum

$$
f=l+g \text { where } l \text { is linear and } g \text { is odd. }
$$

A surprising counter-example due to Hille and Szasz and to Ching (see section 3 below) shows that there are continuous functions not of this form for which $\mathscr{T}$ is exact. In this paper we show that if $\mathscr{T}$ is exact for a function $f$ then $f$ has the form (1) provided it has either continuous second derivative or absolutely convergent Fourier series.

Our results are also pertinent to the general question: how much information about $f$ is contained in the sequence $\left(T_{n} f\right)_{n}$ of approximate integrals of $f$ ? By contrast with, say, the Fourier transform $\hat{f}$, one expects to get very little information. It is perhaps surprising that, when $f$ has either continuous second derivative or absolutely convergent Fourier series, the sequence $\left(T_{n} f\right)_{n}$ actually determines $f$ to within a periodic sine series (see Corollary 1).

To aid our study of functions for which $\mathscr{T}$ is exact we say that a continuous function $f$ lies in the kernel of $\mathscr{T}$ if

$$
T_{n} f=0 \text { for } n=1,2,3, \ldots
$$

Define a function to be odd if, for each $x \in[0,1], f(x)+f(1-x)=0$. This coincides with the usual definition when $f$ is periodic on the real line with period one, for then $f(-x)=f(1-x)$ and so our definition says $f(x)=-f(-x)$. Throughout the paper $C(0,1)$ denotes the space of all continuous, complex-valued functions $f$ on $[0,1]$ which are periodic in the sense that $f(0)=f(1)$. For an integer $n$, the $n$th Fourier coefficient of $f \in C(0,1)$ is given by

$$
f(n)=\int_{0}^{1} f(x) e^{-2 \pi i n x} d x .
$$

We define $A(0,1)$ to be the space of all functions $f \in C(0,1)$ having absolutely convergent Fourier series:

$$
\sum_{\mathbf{z}}|\hat{f}|<\infty
$$

With this notation, our central result (Theorem 1) is: if $f \in A(0,1)$ and $f$ lies in the kernel of $\mathscr{T}$ then $f$ is odd. This extends several results of Ching and Chui $([1,2,3,4])$ which may, with superficial changes, be summarized as follows: if $f \in C(0,1)$ and $f$ lies in the kernel of $\mathscr{T}$ then $f$ is odd provided one of the following conditions is satisfied:

(a) $f^{\prime} \in H^{1}$;

(b) $\hat{f}(n)=O\left(n^{-(1+\varepsilon)}\right)$ for some $\varepsilon>0$;

(c) $\sum_{|n| \geqslant N}|\hat{f}(n)|=O\left(N^{-1}\right)$; or

(d) $f(x)=\sum_{n \geqslant 0} a_{n} e^{2 \pi i q^{n} x}$ where $q$ is a positive integer. 
In each case above $f \in A(0,1)$. For (a) this follows by Hardy's inequality (see [6], page 49); for (b) and (c) it is obvious; and moreover (d) is true whenever $\left\{q^{n}: n \geqslant 0\right\}$ is replaced by any Sidon set (see [7], Chapter 15). However, it is easy to construct elements of $A(0,1)$ satisfying none of the conditions (a) to (d). On the other hand, the counter-example mentioned above shows that some condition is necessary on $f$ for our Theorem 1 to work.

We consider non-periodic functions in Theorem 2 and prove that if $f^{\prime \prime}$ is continuous on $[0,1]$ and $f$ lies in the kernel of $\mathscr{T}$ then $f$ is odd.

We make the following comments about our method of proof. All the cases above due to Ching and Chui stem from a classical Möbius inversion theorem (from [8], page 237). We have found an improved inversion theorem (see [10]) and this leads to a quick proof of Theorem 1 below. However, in this paper, we give a self-contained proof which we exploit in section 3 to characterize those sequences arising as $\left(T_{n} f\right)_{n}$ with $f \in A(0,1)$.

In section 4 we consider one-sided versions of Theorem 1 in which $T_{n} f=0$ is replaced by $T_{n} f \geqslant 0$, and give counter-examples to the obvious conjectures.

As justification for considering only continuous functions in this paper, we envisage $f$ as being unknown except for its values tabulated at the points of evaluation of $\mathscr{T}$; (for finitely many $T_{n}$ this table may be the outcome of an experiment). In this case continuity is the most natural assumption which rules out pathological extensions of the given data which are possible if the Lebesgue integral is used. Indeed, $\mathscr{T}$ depends upon a set of points of evaluation which is countable and so has Lebesgue measure zero. Hence arbitrary extensions are possible if non-continuous functions are considered and then the $T_{n} f$ give no information about the Lebesgue integral of $f$.

We observe that different rules of quadrature seem to need quite different techniques, and hope to return to these in a later paper; see [10].

\section{Main results}

To prove our main results we pass to the Fourier transform. In this context we have:

LeMma 1. Iff $\in C(0,1)$ then $f$ is odd if and only if $f$ is odd in the usual sense that, for each integer $n, \hat{f}(-n)+\hat{f}(n)=0$.

Proof. The $n$th Fourier coefficient of the function $f(x)+f(1-x)$ is $\hat{f}(-n)+\hat{f}(n)$. So, by the uniqueness theorem ([7], section 2.4.1), $f$ is odd if and only if $\hat{f}$ is odd.

Next we recast the kernel of $\mathscr{T}$ in terms of Fourier series. We write $\mathbf{N}$ for the positive integers and $n \mathbf{Z}$ for the subgroup $\{n j: j \in \mathbf{Z}\}$ of the integers $\mathbf{Z}$. 
LEMma 2. If $f \in A(0,1)$ then, for each $n \in \mathrm{N}, T_{n} f=\sum_{n} \mathrm{z} \hat{f}$. If $f$ merely belongs to $C(0,1)$, then $\sum_{n \mathrm{z}} \hat{f}$ is Cesàro summable to $T_{n} f$.

Proof. Firstly, we observe that, if $k \in \mathbf{Z}$ and $e_{k}$ denotes the function $x \mapsto e^{2 \pi / k x}$ on $[0,1]$, then, since $e_{k}$ is periodic,

where $\alpha=e^{2 \pi i k / n}$. Thus

$$
T_{n}\left(e_{k}\right)-n^{-1} \sum_{j=1}^{n} x^{j}
$$

$$
T_{n}\left(e_{k}\right)= \begin{cases}0 & \text { if } n \times k \\ 1 & \text { if } n \mid k\end{cases}
$$

Next, if the $N$ th Cesàro sum of $f$ is given by

then by linearity

$$
\sigma_{N} f=\sum_{|k| \leqslant N}\left(1-\frac{|k|}{N+1}\right) \hat{f}\left(k i e_{k}\right.
$$

$$
T_{n}\left(\sigma_{N} f\right)=\sum_{|k| \leqslant N, k \in n \mathbf{Z}}\left(1-\frac{|k|}{N+1}\right) \hat{f}(k) .
$$

Now as $N$ increases, $\sigma_{N} f$ tends uniformly to $f([7]$, section 6.1 .1$)$; hence, by continuity of $T_{n}, T_{n}\left(\sigma_{N} f\right) \rightarrow T_{n} f$ and so the second assertion follows.

The first assertion is proved by applying the argument above directly to the unweighted partial sums of the Fourier series of $f$.

Our main result is:

THEOREM 1. If $f \in A(0,1)$ and $f$ lies in the kernel of $\mathscr{T}$ then $f$ is odd. In fact, $f$ is $a$ (periodic) sine series.

Proof. This is a particular instance of a more general theorem from number theory which we proved in [10]. However, we now give a different, self-contained proof which we use in section 3. From the lemmas we have to show that, if $\sum_{z}|\phi|<\infty$ and $\sum_{n z} \phi=0$ for $n \in \mathbf{N}$, then

$$
\phi(-k)+\phi(k)=0 \quad \text { for } k=0,1,2, \ldots
$$

Our proof can be motivated as follows.

Suppose $\left(a_{n}\right)_{n \geqslant 0}$ is a one-sided sequence. Ignoring the question of convergence, cancellation gives

$$
\begin{aligned}
a_{n}= & \left(a_{n}+a_{2 n}+a_{3 n}+a_{4 n}+\ldots\right) \\
& -\left(a_{2 n}+a_{4 n}+a_{6 n}+a_{8 n}+\ldots\right) \\
& -\left(a_{3 n}+a_{6 n}+a_{9 n}+a_{12 n}+\ldots\right) \\
& +\left(a_{6 n}+a_{12 n}+a_{18 n}+a_{24 n}+\ldots\right)
\end{aligned}
$$


To deal with the progressions on the right we let $\xi_{n}$ denote the characteristic function of $n \mathbf{Z}$. The distribution of plus and minus signs which gives the right cancellation is defined by the Möbius function:

and

$$
\begin{aligned}
& \mu(1) \quad=1, \\
& \mu\left(p_{1} p_{2} \ldots p_{k}\right)=(-1)^{k} \quad \text { if } p_{1}, p_{2}, \ldots, p_{k} \text { denote distinct primes }
\end{aligned}
$$

$\mu(n) \quad=0 \quad$ if $n$ has a squared prime factor.

We let $\mathbf{P}$ denote the set of primes together with 1 .

To get convergence in the scheme above we define a sequence of functions $\left(\psi_{p}\right)_{p \in \mathbf{P}}$ by

$$
\begin{aligned}
\psi_{1} & =\xi_{1}, \\
\psi_{2} & =\xi_{1}-\xi_{2}, \\
\psi_{3} & =\xi_{1}-\xi_{2}-\xi_{3}+\xi_{6}, \\
\vdots & \\
\psi_{p} & =\sum_{n \in \Pi_{p}} \mu(n) \xi_{n},
\end{aligned}
$$

where $\Pi_{p}=\left\{p_{1} p_{2} \ldots p_{k}: p_{j} \leqslant p\right.$ for $1 \leqslant j \leqslant k$ and $\left.k \in \mathrm{N} \cup\{0\}\right\}$. Now

$$
\psi_{p}(j)=\sum_{n \in \Pi_{p}, n \mid j} \mu(n)= \begin{cases}1 & \text { if all prime factors of } j \text { exceed } p \\ 0 & \text { otherwise }\end{cases}
$$

(see [8], section 16.3). Secondly, $\psi_{p}(j)$ tends to 0 if $|j| \neq 1$, and to 1 if $|j|=1$. Thirdly, $\sum_{\mathbf{z}} \psi_{p} \phi=0$ for each $p \in \mathbf{P}$ since, by hypothesis, $\sum \xi_{n} \phi=0$ for $n \in \mathbf{N}$.

Consequently $\psi_{n} \phi$ is absolutely summable over $\mathbf{Z}$, is dominated by $|\phi|$, and $\psi_{n} \phi$ tends pointwise to $\phi$ times the characteristic function of $\{-1,1\}$. So by the dominated convergence theorem for $l^{1}(Z)$,

$$
\sum_{\mathbf{Z}} \psi_{p} \phi \rightarrow \phi(-1)+\phi(1)
$$

and so the latter is zero.

If $k>1$, we replace $\mathbf{Z}$ in the argument above by $k \mathbf{Z}$ to deduce $\phi(-k)+\phi(k)=0$. Finally, using the initial hypothesis again, $\phi(0)=0$.

Thus if $f \in A(0,1)$ lies in the kernel of $\mathscr{T}$ then, for each $k, \hat{f}(-k)+\hat{f}(k)=0$, and so $f(x)=\sum_{n \in \mathrm{N}} \hat{f}(n) \sin (2 \pi n x)$.

We are indebted to Professor I. H. Sloan for suggestions which lead us to extend the previous result to non-periodic functions in the following way:

THEOREM 2. If $f^{\prime \prime}$ is continuous on $[0,1]$ and $f$ lies in the kernel of $\mathscr{T}$ then $f$ is odd. 
Proof. Firstly, we can find an odd polynomial $p$ such that $f-p \in C^{2}(0,1)$ (This notation, in line with our definition of $C(0,1)$, means that the first two derivatives of $f$ are periodic as well as continuous.) Indeed, letting

$$
p(x)=a\left(x-\frac{1}{2}\right)+b\left(x-\frac{1}{2}\right)^{3}+c\left(x-\frac{1}{2}\right)^{5},
$$

$\Gamma$ is clearly odd whatever coefficients are chosen; we determine them by insisting that

$$
p^{(k)}(1)-p^{(k)}(0)=f^{(k)}(1)-f^{(k)}(0) \text { for } k=0,1,2 .
$$

An easy computation shows that these three equations can be solved for $a, b, c$; let us take this as done. It means that the function $g \equiv f-p$ and its first two derivatives are periodic, as claimed.

Since $g \in C^{2}(0,1)$,

$$
\hat{g}(n)=O\left(n^{-2}\right),
$$

and so $g \in A(0,1)$. Since $p$ is odd, $g$ lies in the kernel of $\mathscr{T}$. So, by Theorem 1 , $g$ is odd. Again, since $p$ is odd, $f$ must be odd.

From these theorems we have two easy consequences.

COROLlaRY 1. If $T_{n} f=T_{n} g$ for each $n \in \mathbf{N}$ and $f, g$ have either absolutely convergent Fourier series or continuous second derivative, then $f$ and $g$ differ by an odd function.

Concerning exactness of $\mathscr{T}$ we have:

COROLlARY 2. If $\mathscr{T}$ is exact for $f$ and $f$ has either absolutely convergent Fourier series or continuous second derivative, then there is an odd function $g$ such that

$$
f=g+\int_{0}^{1} f
$$

Observe that in all these results it is necessary to assume $T_{n} f=0$ for every $n \in \mathbf{N}$. Indeed, if $m$ is any positive integer, the function

$$
g_{m}(x)=\sum_{j \mid m} \mu(m / j) \cos (2 \pi j x)
$$

is clearly in $A(0,1)$. By Lemma 1 and properties of $\mu$,

$$
T_{n}\left(g_{m}\right)=\sum_{k n \mid m} \mu(m / k n)= \begin{cases}1 & \text { if } m=n, \\ 0 & \text { otherwise }\end{cases}
$$

Nevertheless, $g_{m}$ is an even function. 


\section{Möbius summation}

We can get a little more from the proof of Theorem 1. As before, we let $\mathbf{P}$ denote the set of primes together with 1 and let $\Pi_{p}$ denote the set of square-free positive integers whose prime factors do not exceed $p$.

For $p \in \mathbf{P}$ we define the kernel $\mu_{p}$ by

$$
\mu_{p}(n)= \begin{cases}\mu(n) & \text { if } n \in \Pi_{p} \\ 0 & \text { otherwise }\end{cases}
$$

Also, we take the convolution product of two function $\phi$ and $\psi$ on $\mathbf{N}$ to be

$$
\phi * \psi(n)=\sum_{j \in \mathbb{N}} \phi(j) \psi(j n)
$$

whenever the series converges.

With this natation we can rewrite

$$
\sum_{j \in \Pi_{p}} \mu(j) \sum_{k \in \mathbf{Z}} \phi(j k n)
$$

from the schema in the proof of Theorem 1 as

$$
\mu_{p} * \Phi(n),
$$

where $\Phi(n)=\sum_{n Z} \phi$. The proof shows that if $\phi \in l^{1}(Z)$ then

$$
\mu_{p} * \Phi(n) \rightarrow \phi(-n)+\phi(n) \text { as } p \rightarrow \infty \text {. }
$$

This suggests the following definition:

Definition. Suppose $\Phi$ is a function on $\mathbf{N}$. We say that $\Phi$ is Möbius summable to a function $\psi$ on $\mathbf{N}$ if, for all $n \in \mathbf{N}, \mu_{p} * \Phi(n) \rightarrow \psi(n)$ as $p \rightarrow \infty$.

By Theorem 1 and the uniqueness theorem for Fourier transforms, we have:

LEMma 3. If $f \in A(0,1)$ then $\left(T_{n} f\right)_{n}$ is the unique sequence $\Phi$ of the form $\Phi(n)=\sum_{n Z} \phi$ with $\phi \in l^{1}(\mathbf{Z})$, for which $\mu_{p} * \Phi$ tends pointwise to the Fourier transform of the function $f(x)+f(1-x)$.

This enables us to identify the space $\mathscr{T}(A(0,1))$ of all sequences $\left(T_{n} f\right)_{n}$ with $f$ in $A(0,1)$.

TheOREM 3. A sequence $\Phi$ of the form $\Phi(n)=\sum_{n \mathbf{Z}} \phi$ for $\phi \in l^{1}(\mathbf{Z})$ is in $\mathscr{T}(A(0,1))$ if and only if $\Phi$ is Möbius summable to $\phi$.

Proof. By Lemma 3 it suffices to prove the converse implication; suppose $\Phi$ is Möbius summable to $\phi$, and set

$$
f(x)=\sum_{n \in N} \phi(n) \cos 2 \pi n x .
$$


Then $f \in A(0,1)$ and $T_{n} f=\frac{1}{2} \sum_{n Z} \phi$ whence $\left(T_{n} f\right)_{n}$ is Möbius summable to $\phi \in l^{1}(\mathrm{Z})$. By the uniqueness part of Lemma $3, T_{n} f=\Phi(n)$ as required.

Hille and Szasz ([9], page 417) and Ching (in [1], page 25) have considered the following example:

$$
g=\sum_{n \in \mathbf{N}} \frac{\mu(n)}{n} e_{n}
$$

By the estimate of Davenport ([5], Theorem 1), the series is uniformly convergent but, by Lemma $1, g$ is not odd. As easy calculation shows that $g$ lies in the kernel of $\mathscr{T}$.

On the one hand, this example shows that nothing like Theorems 1 and 2 holds for general continuous functions on $[0,1]$. On the other hand, it shows that some condition like the hypothesis of Theorem 2 is necessary to remain inside $\mathscr{T}(A(0,1))$.

\section{One-sided versions}

It is natural to seek a generalization of Theorem 1 (and hence automatically of Theorem 2) with $T_{n} f=0$ replaced by $T_{n} f \geqslant 0$, and a slightly weakened conclusion. In this section we show that two natural conjectures of this type are false, and give two positive results.

Clearly, if $f \in C(0,1)$ satisfies $\hat{f}(-n)+\hat{f}(n) \geqslant 0$ for $n=0,1,2,3, \ldots$ then; from Lemma $1, T_{n} f \geqslant 0$ for each $n \in \mathbf{N}$. The next two examples (and also the one in section 3) show that the converse is false.

Example 1. The function $f=-e_{1}+e_{2}+e_{3}$ belongs to $A(0,1)$ and

$$
\hat{f}(n)=\left\{\begin{aligned}
-1 & \text { if } n=1 \\
1 & \text { if } n=2,3 \\
0 & \text { otherwise }
\end{aligned}\right.
$$

So

$$
T_{n} f= \begin{cases}1 & \text { if } n=1,2,3 \\ 0 & \text { otherwise }\end{cases}
$$

Consequently $T_{n} f \geqslant 0$ for $n \in \mathbf{N}$, and yet $\hat{f}(-1)+\hat{f}(1)<0$.

Example 2. Example 1 is rather trivial because it has only finitely many $n$ for which $T_{n} f$ is nonzero, and only one $n$ for which $\hat{f}(-n)+\hat{f}(n)<0$. Let $\phi \in l^{1}(\mathbf{P})$ be everywhere positive. Set

$$
f=\sum_{\mathbf{p} \in \mathbf{P}} \phi(p)\left(e_{2 p}-e_{p}\right)
$$


Then $f \in A(0,1)$ and

$$
\hat{f}(n)=\left\{\begin{array}{cl}
\phi(0)-\phi(2) & \text { if } n=2 \\
-\phi(n) & \text { if } n \in \mathbf{P} \backslash\{2\} \\
\phi(n / 2) & \text { if } n / 2 \in \mathbf{P} \\
0 & \text { otherwise }
\end{array}\right.
$$

So a straightforward calculation shows that

$$
T_{n} f= \begin{cases}\sum_{p \in \mathbf{P} \backslash\{2\}} \phi(p) & \text { if } n=2 \\ \phi(n / 2) & \text { if } n / 2 \in \mathbf{P} \backslash\{2\} . \\ 0 & \text { otherwise }\end{cases}
$$

Consequently $T_{n} f \geqslant 0$ for each $n \in \mathbf{N}$, yet $\hat{f}(-p)+\hat{f}(p)<0$ for each $p \in \mathbf{P}$.

Alternatively, and unrelated to the condition above, if $f \in C(0,1)$ and $f(x)+f(1-x) \geqslant 0$ for $x \in[0,1]$, then $T_{n} f \geqslant 0$ for $n \in \mathbf{N}$. Again the converse is false.

Example 3. Define

$$
f(x)= \begin{cases}-10 x & \text { if } 0 \leqslant x \leqslant 1 / 10 \\ 10(x-1 / 5) & \text { if } 1 / 10 \leqslant x \leqslant 3 / 10 \\ 1 & \text { if } 3 / 10 \leqslant x \leqslant 7 / 10 \\ -10(x-4 / 5) & \text { if } 7 / 10 \leqslant x \leqslant 4 / 5 \\ 0 & \text { if } 4 / 5 \leqslant x \leqslant 1\end{cases}
$$

Then $f(1 / 10)<0$, yet calculation shows $T_{n} f \geqslant 0$ for each $n \in \mathbf{N}$.

In the positive direction we have two results. In the first $\zeta(s)=\sum_{n \in \mathbb{N}} n^{-s}$.

THEOREM 4. Let $\rho$ be the unique real root of $\zeta(\rho)=2$ with $\rho>1$. If $f \in A(0,1)$, $T_{n} f \geqslant 0$ for each $n \in \mathbf{N}$, and $T_{m n} f \leqslant m^{-\rho} T_{n} f$, for $m, n \in \mathbf{N}$, then, for each $n \in \mathbf{N}$, $\hat{f}(-n)+\hat{f}(n) \geqslant 0$.

Proor. By the inversion process of the proof of Theorem 1,

Now

$$
\hat{f}(n)+\hat{f}(-n)=\sum_{n \in \mathbb{N}} \mu(m) T_{m n} f
$$

hence

$$
\left|\sum_{m \geqslant 2} \mu(m) T_{m n} f\right| \geqslant \sum_{m \geqslant 2} m^{-\rho} T_{n} f=(\zeta(\rho)-1) T_{n} f=T_{n} f
$$

$$
\hat{f}(-n)+\hat{f}(n) \geqslant 0 \text {. }
$$

Our final result shows that, under an extra condition, the assumption $T_{n} f \geqslant 0$ for each $n \in \mathbf{N}$ is sufficient to ensure $f$ is odd. 
TheOrem 5. Let $f \in A(0,1)$ with $T_{n} f \geqslant 0$ for $n \in \mathbf{N}$. Suppose that, for $p \in \mathbf{P}, n \in \mathbf{N}$,

$$
\sum_{s(n, p)} \hat{f} \leqslant 0,
$$

where $S(n, p)=\cup\{n q \mathrm{Z}: q \in \mathbf{P}$ and $2 \leqslant q \leqslant p\}$. Then $f$ is odd.

Proof. If we can show $\sum_{n \mathrm{z}} f \leqslant 0$ for each $n$, then $T_{n} f=0$, and so Theorem 1 applies to yield the conclusion. In fact we do this in an apparently roundabout manner by showing $\hat{f}(-k)+\hat{f}(k) \geqslant 0$; then from $\sum_{s(n, p)} \hat{f} \leqslant 0$ we can conclude $\hat{f}(-k)+\hat{f}(k)=0$.

Suppose initially that $k=1$. An easy manipulation using the properties of the Möbius function yields, in the notation of the proof of Theorem 1, for each $p \in \mathbf{P}$.

$$
\sum_{n \in \Pi_{p}} \mu(n) \sum_{n \mathbf{Z}} \hat{f}=\sum_{\mathbf{Z}} \hat{f}-\sum_{s(1, p)} \hat{f} \geqslant 0 .
$$

(For example, when $p=3$,

$$
\begin{aligned}
\sum_{\mathbf{Z}} \hat{f}-\sum_{\mathbf{2} \mathbf{Z}} \hat{f}-\sum_{\mathbf{3} \mathbf{Z}} \hat{f}+\sum_{\mathbf{6} \mathbf{Z}} \hat{f} & =\sum_{\mathbf{Z}} \hat{f}-\sum_{\mathbf{2} \mathbf{2} \cup 3 \mathbf{Z}} \hat{f}-\sum_{2 \mathbf{Z} \cap \mathbf{3} \mathbf{Z}} \hat{f}+\sum_{\mathbf{6} \mathbf{Z}} \hat{f} \\
& =\sum_{\mathbf{Z}} \hat{f}-\sum_{2 \mathbf{Z} \cup 3 \mathbf{Z}} \hat{f} \\
& \left.=\sum_{\mathbf{Z}} \hat{f}-\sum_{s(1,3)} \hat{f} .\right)
\end{aligned}
$$

So, interchanging sums,

$$
\sum_{\mathbf{Z}} \hat{f} \psi_{p}=\sum_{j \in \mathbf{Z}} \hat{f}(j) \sum_{n \in \Pi_{p}} \mu(n) \xi_{n}(j) \geqslant 0 .
$$

From the proof of Theorem $1, \psi_{p}$ tends pointwise to the characteristic function of $\{-1,1\}$; hence $\hat{f}(-1)+\vec{f}(1) \geqslant 0$.

Finally, taking $k>1$, the same argument shows $\hat{f}(-k)+\hat{f}(k) \geqslant 0$.

\section{References}

[1] G. R. Blakeley, I. Borosh and C. K. Chui, "A two-dimensional mean problem", J. Approx. Theory 22 (1973), 11-26.

[2] C. H. Ching and C. K. Chui, "Uniqueness theorems determined by function values at the roots of unity", J. Approx. Theory 9 (1973), 267-271.

[3] C. H. Ching and C. K. Chui, "Analytic functions characterized by their means on an arc", Trans. Amer. Math. Soc. 184 (1973), 175-183.

[4] C. K. Chui and C. H. Ching, "Approximation of functions by their means", in Symposium on approximation theory (ed. G. G. Lorentz) (New York: Academic Press, 1973), p. 307312.

[5] H. Davenport, "On some infinite series involving arithmetical functions II", Quart. J. Math. 8 (1937), 313-320.

[6] P. L. Duren, Theory of $\boldsymbol{H}^{p}$ spaces (New York: Academic Press, 1970). 
[7] R. E. Edwards, Fourier series: a modern introduction Vols I and II (New York: Holt, Rinehart and Winston, 1967).

[8] G. H. Hardy and E. M. Wright, An introduction to the theory of numbers (Oxford: Oxford University Press, 1965).

[9] E. Hille and O. Szasz, "On the completeness of Lambert functions", Bull. Amer. Math. Soc. 42 (1936), 411-418.

[10] J. H. Loxton and J. W. Sanders, "On an inversion theorem of Möbius" submitted to $J$. Austral. Math. Soc., Series A.

School of Mathematics

University of New South Wales

Kensington

N.S.W. 2033 